\title{
Non-destructive evaluation of the spatial variability of reinforced concrete structures
}

\author{
Ngoc Tan Nguyen ${ }^{a}$, Zoubir Mehdi Sbartaï, Jean-François Lataste, Denys Breysse \\ AND FRÉDÉRIC BOS
}

University of Bordeaux, I2M, UMR 5295, 33400 Talence, France

Received 17 July 2013, Accepted 5 July 2014

\begin{abstract}
Spatial variability of concrete is an important characteristic, which qualifies the nonhomogeneity of concrete properties within structural components. Assessing it can be of great interest for damaged areas localization and for reliability analysis of structures. A two-stage experimental program was carried out using non-destructive testing (NDT) on laboratory concrete slabs in outdoor environment and on an existing bridge. The spatial variability was quantified at three scales: point (repeatability), local and global, for several NDT techniques (ultrasonic pulse velocity, electrical resistivity, radar and rebound hammer). The experimental results were analyzed using statistical and geostatistical tools.
\end{abstract}

Key words: Concrete / non-destructive testing / spatial variability / uncertainty / spatial correlation / variogram

\section{Introduction}

The spatial variability of concrete is an important characteristic, which qualifies the non-homogeneity of mechanical and physical properties on structural components. It derives from the intrinsic variability of composition (e.g. size and nature of aggregates), the environmental conditions during the life of the structure (e.g. temperature and humidity) and the implementation conditions (e.g. workmanship). Assessing the spatial variability of concrete structures can be of major interest for either locating potential damaged areas in an existing structure, or reliability analysis.

Theoretically, it is possible to assess the spatial variability by destructive method, but this is very difficult in practice and impossible in many situations, since it requires a high number of cores. It is a reason why nondestructive testing (NDT) techniques have been considered as an interesting tool for providing access to material properties while maintaining moderate cost [1]. Several NDT have been used to assess the variability of concrete in the framework of French research projects [2,3]. However, most of studies have been performed on specimens in the laboratory. The spatial variability is rarely evaluated on existing structures. In fact, electrical resistivity and radar techniques are sensitive to water content [4],

\footnotetext{
${ }^{a}$ Corresponding author:

ngoc-tan.nguyen@u-bordeaux.fr
}

chloride [5,6], and porosity [7]. Ultrasonic and rebound hammer techniques are widely used for mechanical properties evaluation such as strength as well as Young's modulus [1]. The simultaneous use of these techniques can provide an efficient evaluation of the spatial variability of concrete structures.

On one hand, assessing the spatial variability is often limited to scatter estimation. The material property is considered as a random variable, whose value at a specific point can be seen as the realization of a random experiment. In this case, the spatial variability is often quantified by the coefficient of variation of a measurement set, which is standard deviation divided by the mean. On the other hand, recent reliability studies have shown that the spatial correlation may govern the reliability of structural components [8]. It is considered that the values taken by the material property at two close points can be very close, and that the difference increases when two points are taken at some distance. In the second case, assessing the spatial variability is based on the sample variance at some specific distance, named the correlation length. First studies have been devoted to the estimation of the spatial correlation of NDT measurements $[9,10]$.

This paper analyzes recent and original works on the concrete variability evaluation using NDT techniques. An outdoor site-test was first implemented in the laboratory. This site-test was made of five reinforced concrete slabs of $2 \times 2.45 \times 0.2 \mathrm{~m}^{3}$ with different strengths ranging from 25 to $45 \mathrm{MPa}$. The concrete slabs have been designed with 
characteristics of existing structures such as dimensions, strength, concrete cover, etc. The methodology consists of performing statistical analysis for assessing the spatial variability and estimating the required minimal number of NDT measurements at three scales: point (repeatability), local and global. According to the laboratory results, NDT measurements were also carried out on the piers of a bridge as an on-site case study, focusing on the estimation of mechanical strength.

\section{Assessing material variability on model slabs}

\subsection{Experimental program: outdoor site-test and NDT}

A large experimental program was conducted using NDT techniques on a site-test in outdoor environment. This testing site was made of five reinforced concrete slabs of $2 \times 2.45 \times 0.2 \mathrm{~m}^{3}$. The slabs were cast with industrial concretes of different strengths ranging from 25 to $45 \mathrm{MPa}$. The rebar grid has a regular spacing of $45 \mathrm{~cm}$, and is made of HA10 and HA14 steel bars. This grid serves as a basis for the analysis of variability: the $45 \times 45 \mathrm{~cm}^{2}$ area is considered as a testing area. For each concrete mix, 12 cubic specimens of $15 \times 15 \times 15 \mathrm{~cm}^{3}$ were manufactured and stored under the same exposure conditions as the slabs [11]. The specimens are used for the destructive tests (compression test, porosity, water content) at the same time (day) as NDT measurements. Several NDT were also carried out on the specimens in order to compare them with those on the slabs.

Four NDT techniques have been used:

1. ultrasonic pulse velocity (UPV) measurements were performed by both modes: direct and indirect transmission of the propagation of ultrasonic waves at $200 \mathrm{kHz}$ frequency;

2. electrical resistivity measurements were performed using a four-probe device adapted to on-site investigations [12]. The result is given in terms of apparent resistivity. This technique allows the assessment of near surface material properties;

3. radar (Ground Penetrating Radar - GPR) measurements were performed on the slabs by the GSSI SIR3000 system. An antenna of $2.6 \mathrm{GHz}$ frequency was continuously moved on the slab surface with a regular spacing between profiles, along longitudinal and transversal directions;

4. rebound hammer measurements were performed on three different sides (lateral, bottom, top) of each specimen, and at 16 test points for each side with a regular spacing of $3 \mathrm{~cm}$. On the slabs, 36 measurements were performed with a regular spacing of $5 \mathrm{~cm}$ in each testing area.

Then the spatial variability was quantified at three scales:

- Variability $V_{R}$ results from the imperfect repeatability of measurements, which corresponds to the measurement error due to the equipment and contact between sensor and concrete surface. $V_{R}$ was estimated by repeating 20 times the same measurement at a given point in the course of a few minutes. This is not possible with rebound, since the hammer damages slightly the surface of testing point.

- Variability $V_{L}$ results from the internal variability of the material at local scale (small distance), for example, within a reinforcing mesh or the specimen size. The local variability represents the irreducible constitutive non-homogeneity of the material (e.g. that due to spatial distribution of aggregates or surface roughness). $V_{L}$ was estimated in each testing area (reinforcing mesh or specimen surface).

- Variability $V_{G}$ results from the variability of the material at global scale (large distance) i.e. on many testing areas within a slab. Each slab has nine or fifteen testing areas. These areas were evenly distributed over the surface of the slabs. Then the global variability is estimated by all measurements.

This enables uncertainty evaluation (making the part between what is due to non-perfect measurements and what comes from real material variability) and estimation of the minimum number of measurements in order to assess the material properties with a given level of confidence.

\subsection{Experimental results: magnitude of variability and spatial correlation}

The statistical results are summarized in Table 1 for three concrete mixes (D1, D3, and D5 slabs) and their specimens, noted SP. The measured parameters are the compressive strength $\left(f_{\mathrm{c} 28}\right)$ in MPa at 28 days, direct and indirect UPVs in $\mathrm{m} . \mathrm{s}^{-1}$, electrical resistivity $\left(\rho_{a}\right)$ in $\Omega . \mathrm{m}$, rebound index, and GPR positive amplitude of direct signal. Table 1 specifies the mean (m) and the coefficient of variation $(\mathrm{CV}$, in \%). Two parameters are used:

- The first one quantifies the overall variability at the slab/specimen scale $\left(V_{G} / V_{S P}\right)$.

- The second one is the mean value of all local variabilities calculated on all testing areas $\left(V_{L}\right)$.

When comparing the NDT variability between these slabs, the $C V$ values range from 2.8 to $5.1 \%$ for UPV, from 7.2 to $8.6 \%$ for rebound and from 4.7 to $5.6 \%$ for GPR positive amplitude. The electrical resistivity is more variable, which ranges from $5.1 \%$ to $12.2 \%$. The magnitude of $C V$ seems to be a little lower for the D5 slab having the highest strength. The D1 slab has intermediate values. As a consequence, the D1 slab was selected to discuss further the variability in the following. In all cases, the global variability $V_{G}$ of the slabs is larger than the average value of the local variability $V_{L}$.

In the next step, the concrete properties are studied as being spatially correlated using geostatistical tools. The variogram is a practical mean for describing the spatial correlation. The experimental variogram at the lag distance $h$ is computed by Equation (1) with $N$ being the 
N.T. Nguyen et al.: Mechanics \& Industry 16, 103 (2015)

Table 1. NDT variability of concretes at 28 days: mean and coefficient of variation.

\begin{tabular}{|c|c|c|c|c|c|c|c|c|c|c|}
\hline \multirow{2}{*}{ Slab } & \multirow{2}{*}{$f_{c 28}[\mathrm{MPa}]$} & \multirow{2}{*}{ Scale } & \multicolumn{2}{|c|}{$\mathrm{UPV}\left[\mathrm{m} \cdot \mathrm{s}^{-1}\right]$} & \multicolumn{2}{|c|}{$\rho_{a}[\Omega . \mathrm{m}]$} & \multicolumn{2}{|c|}{ Rebound } & \multicolumn{2}{|c|}{ GPR } \\
\hline & & & $m$ & $C V$ & $m$ & $C V$ & $m$ & $C V$ & $m$ & $C V$ \\
\hline \multirow{3}{*}{ D1 } & \multirow{3}{*}{26.6} & $\overline{V_{G}}$ & 3632 & 4.2 & 37.7 & 8.4 & 30.3 & 7.2 & 20495 & 5.6 \\
\hline & & $\overline{V_{L}}$ & & 3.3 & & 5.1 & & 6.7 & & 3.7 \\
\hline & & $V_{S P}$ & 4239 & 2.7 & 188.2 & 8.7 & 31.4 & 6.4 & & \\
\hline \multirow{3}{*}{ D3 } & \multirow{3}{*}{37.3} & $V_{G}$ & 3792 & 5.1 & 56.9 & 12.2 & 33.2 & 7.9 & 18439 & 5.4 \\
\hline & & $\overline{V_{L}}$ & & 4.3 & & 6.8 & & 7.5 & & 4.2 \\
\hline & & $V_{S P}$ & 4412 & 1.6 & 130.7 & 10.7 & 34.5 & 8.0 & & \\
\hline \multirow{3}{*}{ D5 } & \multirow{3}{*}{45.6} & $V_{G}$ & 4006 & 2.8 & 68.0 & 5.1 & 37.3 & 8.6 & 21926 & 4.7 \\
\hline & & $\overline{V_{L}}$ & & 2.6 & & 3.7 & & 8.4 & & 3.7 \\
\hline & & $V_{S P}$ & 4534 & 1.3 & 133.5 & 6.1 & 41.9 & 6.7 & & \\
\hline
\end{tabular}

number of pairs for lag $h ; z$ is the measured value at the location $u$ in the study area.

$$
y(h)=\frac{1}{2 N(h)} \sum_{N(h)}[z(u)-z(u+h)]^{2}
$$

In practice, for the variogram to be reliable, lag cannot be larger than half the size of the study area [13]. For example, the experimental variograms of UPV and electrical resistivity are plotted in Figure 1 for the D1 slab. These variograms were built for a maximal lag of $100 \mathrm{~cm}$, which is half of the slab width. On the same graphs, experimental values (drawn from measurements) and a model curve are superimposed. When analyzing variograms, three characteristics are usually discussed, namely the range, the sill and the nugget. The range is the lag distance at which the measured values are uncorrelated (independent). In this case, it can be named the correlation length of NDT measurements. The sill is the variance value at which the variogram levels off. In some case, the maximal value of variogram is used as information corresponding to the global variability. The nugget quantifies the variability at distances smaller than the minimal spacing of measurements, including the measurement error.

The UPV variogram shows that the measured values are generally uncorrelated (independent). The maximal variance of this variogram is equal to $23141 \mathrm{in} \mathrm{m}^{2} \cdot \mathrm{s}^{-2}$ at the lag of $70 \mathrm{~cm}$. This value infers a variation of $152 \mathrm{~m} . \mathrm{s}^{-1}$, which corresponds to the global variability of UPV measurements on the D1 slab. The nugget effect is equal to 17570 in $\mathrm{m}^{2} \cdot \mathrm{s}^{-2}$. This value shows that the local variability of UPV is about $133 \mathrm{~m} . \mathrm{s}^{-1}$, including the measurement error. The electrical resistivity variogram can be modeled by a spherical model with a specific range of $60 \mathrm{~cm}$ and a sill of $9.1 \mathrm{in} \mathrm{ohm}^{2} \cdot \mathrm{m}^{2}$. This sill infers a $C V$ of $8 \%$ regarding the mean value, which corresponds to the global variability of electrical resistivity measurements on the D1 slab. The first points of this variogram present a high correlation of measurements. The nugget effect is equal to $0.8 \mathrm{in} \mathrm{ohm}^{2} \cdot \mathrm{m}^{2}$. This value shows that the local variability of electrical resistivity is about $2.4 \%$ at distances smaller than $5 \mathrm{~cm}$, including the measurement error.

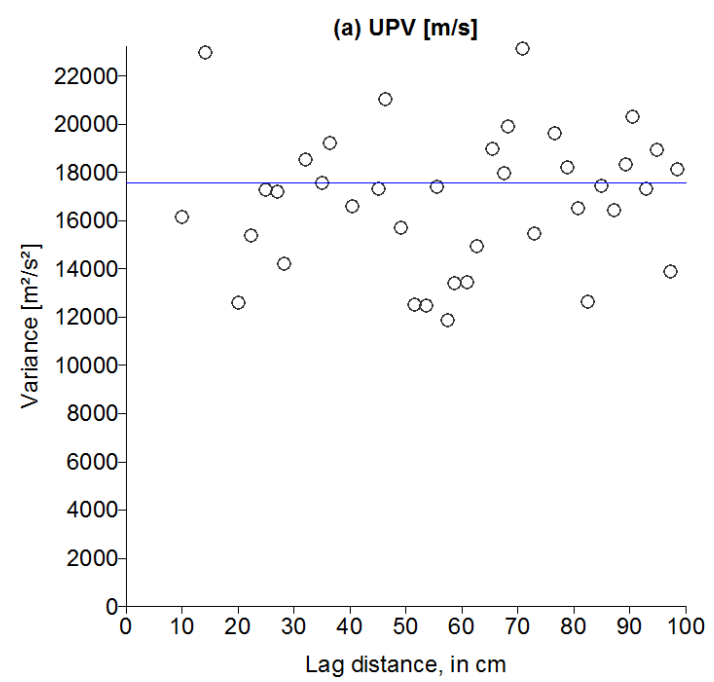

(b) Electrical Resistivity [ohm.m]

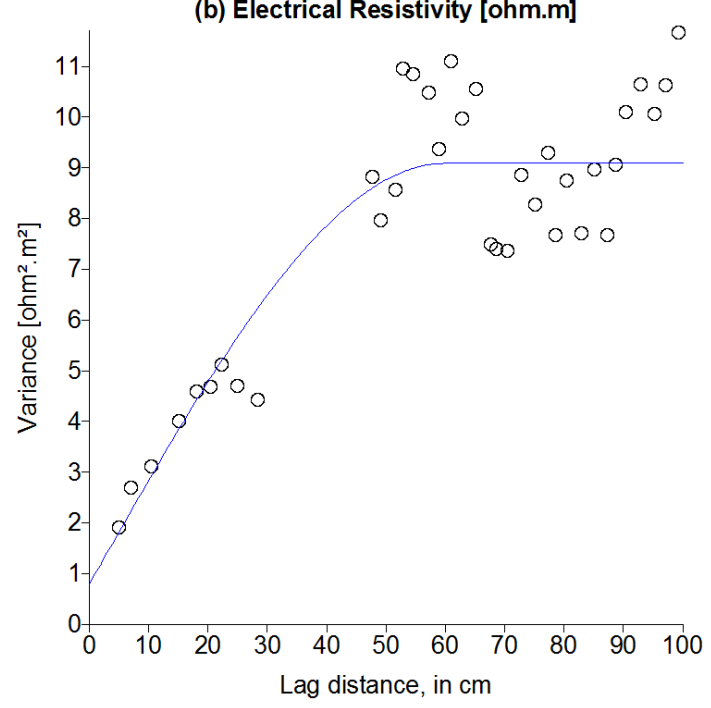

Fig. 1. Experimental and model variograms: (a) UPV, (b) electrical resistivity.

\subsection{Estimation of the minimum numbers of measurements}

The minimum number of measurements is calculated on the basis of $C V$ values corresponding to each specific 
N.T. Nguyen et al.: Mechanics \& Industry 16, 103 (2015)

Table 2. $C V$ values and required numbers of measurements on the D1 slab.

\begin{tabular}{ccccccc}
\hline Technique & $C V_{V_{R}}(\%)$ & $C V_{V_{L}}(\%)$ & $C V_{V_{G}}(\%)$ & $N_{V_{R}}$ & $N_{V_{L}}$ & $N_{V_{G}}$ \\
\hline UPV & 0.3 & 2.8 & 4.2 & 1 & 3 & 4 \\
Electrical resistivity & 0.6 & 4.9 & 8.4 & 1 & 5 & 13 \\
Rebound hammer & - & 7.1 & 7.2 & 1 & 9 & 10 \\
GPR & 0.6 & 4.1 & 5.6 & 1 & 4 & 6 \\
\hline
\end{tabular}

survey $[1,12,14]$. Supposing that the set of measurements $\left(x_{1}, \ldots, x_{n}\right)$ is a sample from a normal distribution with unknown mean $\mu$ and unknown variance $\sigma^{2}$, the confidence interval for $\mu$ with $100(1-\alpha)$ percent confidence denotes as follows:

$$
\mu \in\left[\bar{x}-t_{1-\alpha / 2, n-1} \frac{s}{\sqrt{n-1}}, \bar{x}+t_{1-\alpha / 2, n-1} \frac{s}{\sqrt{n-1}}\right]
$$

where $\bar{x}$ is the observed mean, $s$ is the estimated standard deviation, $n$ is the sample size (measurement number), $t_{1-\alpha / 2, n-1}$ is obtained from $t$-distribution. Hence, the measurement number for estimating the measured average within $\varepsilon$ percent accuracy can be derived as:

$t_{1-\alpha / 2, n-1} \frac{C V}{\sqrt{n-1}} \leqslant \varepsilon \quad \Rightarrow \quad n \geqslant\left(t_{1-\alpha / 2, n-1} \frac{C V}{\varepsilon}\right)^{2}+1$

with $C V$ (in \%) being the coefficient of variation for a set of measurements. The $C V$ values of NDT measurements on the D1 slab are presented in Table 2. With each NDT technique and level of confidence being given, Equation (3) provides the relationship between a target accuracy $\varepsilon$ and the resulting minimum number of measurements, or reversely, the accuracy $\varepsilon$ corresponding to a given number of measurements.

With $95 \%$ confidence interval for the mean evaluation and 5\% accuracy, the minimum numbers are also summarized in Table 2 at three scales (repeatability, local, global), for four NDT techniques. At the local scale, it is at least 3 for UPV, 5 for electrical resistivity, 9 for rebound hammer and 4 for GPR positive amplitude. At the global scale, these numbers increase to 4, 13, 10 and 6 measurements respectively. These values are used when considering the independent measurements. If the spatial correlation of measurements is identified at the global scale, the testing points will be distributed with a sampling distance of at least the correlation length in order to optimize the strategy of diagnosis. For example, 13 measurements of electrical resistivity can be carried out on the D1 slab with a spacing of $60 \mathrm{~cm}$.

\section{Assessing material variability on a RC bridge}

\subsection{Bridge description and NDT}

Within the framework of the C2D2-ACDC French collaborative project, a highway bridge was selected to perform many NDT measurements on an existing structure.
This bridge is located near Valenciennes, in North of France. It is made of three parallel bridges oriented EastWest, laying on a series of sixteen 6-meter high trapezoidal piers. The pier width increases from $1.72 \mathrm{~m}$ at the bottom to $2.9 \mathrm{~m}$ at the top. The pier thickness is $50 \mathrm{~cm}$.

We will focus here on the analysis of UPV and rebound measurements. The spatial variability of the concrete has been evaluated on a testing area of $200 \times 100 \mathrm{~cm}^{2}$ on a bridge pier, which has a regular reinforcing mesh of $25 \times 25 \mathrm{~cm}^{2}$. This pier had been identified by preliminary measurements as the most contrasted among all piers. According to the laboratory results, four UPV measurements with spacing of $7 \mathrm{~cm}$, and twelve rebound measurements with spacing of $5 \mathrm{~cm}$ were performed respectively in each reinforcing mesh area. Thus there were a total of 120 UPV measurements and 288 rebound measurements.

\subsection{Experimental results}

In the first step, the statistical analysis of measurements shows that $90 \%$ of UPV values range between $4070 \mathrm{~m} . \mathrm{s}^{-1}$ and $4667 \mathrm{~m} . \mathrm{s}^{-1}$ (Fig. 2), and $90 \%$ of rebound values range between 44 and 55 . The $C V$ values are also calculated to be $3.7 \%$ with a mean of 4389 m.s ${ }^{-1}$ for UPV and $7.2 \%$ with a mean of 50.4 for rebound. The results show the normal distribution for both techniques.

In the second step, the concrete properties of the bridge pier are assumed as spatially correlated with variograms. The experimental variogram of UPV is also plotted in Figure 2. This variogram is modeled by the spherical model (Eq. (4)) with a specific range of $40 \mathrm{~cm}$ and a sill of $23500 \mathrm{in} \mathrm{m}^{2} \cdot \mathrm{s}^{-2}$. This sill infers a variation of $153 \mathrm{~m} . \mathrm{s}^{-1}$, which corresponds to the global variability of UPV measurements. The nugget effect is equal to 10000 in $\mathrm{m}^{2} \cdot \mathrm{s}^{-2}$. This value shows that the local variability of UPV is about $100 \mathrm{~m} . \mathrm{s}^{-1}$ at distances smaller than $7 \mathrm{~cm}$, including the measurement error.

$$
y(h)=\left\{\begin{array}{lll}
C_{0}+C\left[1.5 \frac{h}{a}-0.5\left(\frac{h}{a}\right)^{3}\right] & \text { if } & h \leq a \\
C_{0}+C & \text { if } & h>a
\end{array}\right\}
$$

where $a$ is the range, $C_{0}$ is the nugget effect, $C_{0}+C$ is the sill, and $h$ is the lag distance.

The main difference with the study on the slabs is that some spatial correlation is here clearly visible, even for UPV measurements; with a nugget which is about half the sill value. The range, distance at which the values can be considered as uncorrelated is about 40 to $60 \mathrm{~cm}$. This distance is important, since it provides an indication of how to carry out investigations if one wants to assess the 

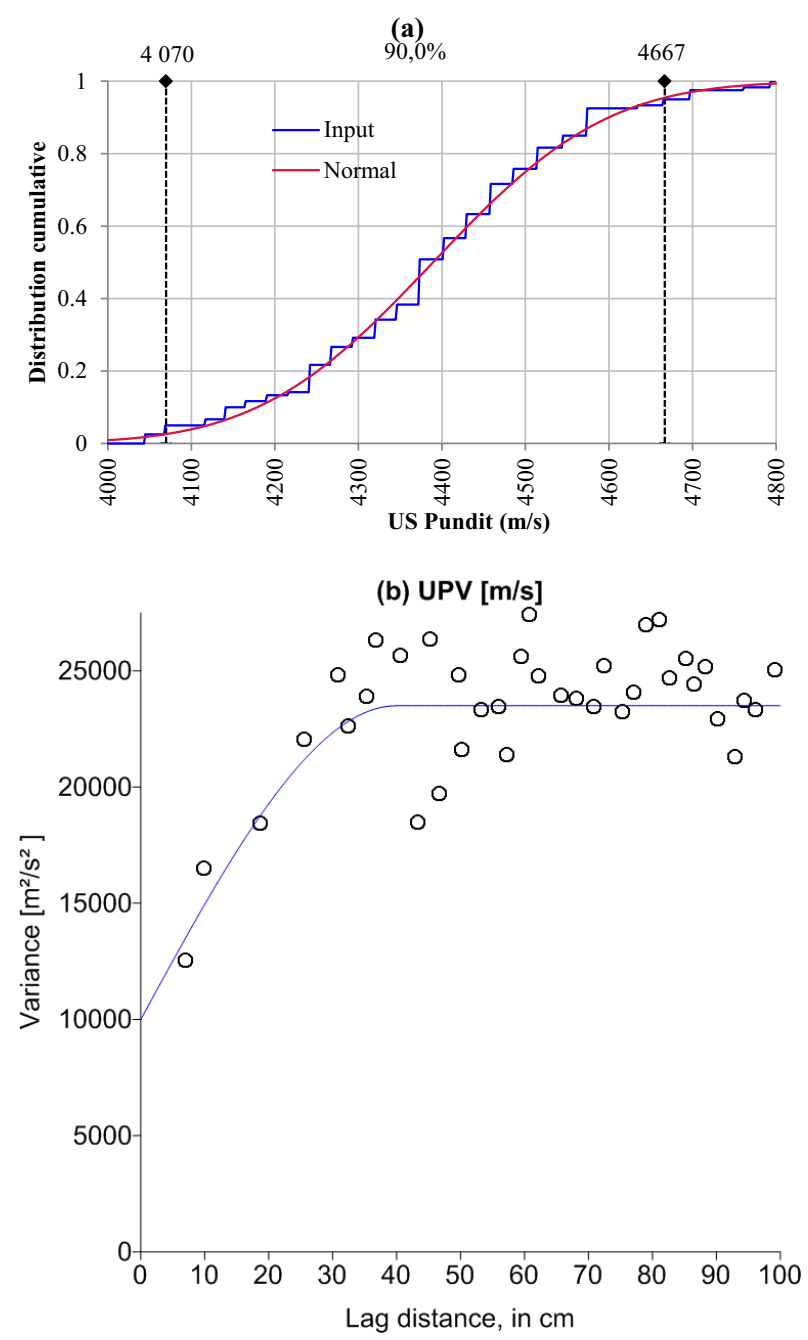

Fig. 2. (a) Cumulative distribution and (b) variogram of UPV.

pier properties. Using this distance and the $C V$ value, the minimum number of NDT measurements is required at least 6 for a testing area of $2 \mathrm{~m}^{2}$. In the case of the investigated pier, three measurements per $\mathrm{m}^{2}$ are required for $2 \%$ accuracy of mean value.

In the next step, the ordinary Kriging was used and two maps were built for (a) UPV and (b) rebound (Fig. 3). Both maps highlight the non-homogeneity of the concrete on the investigated area. A significant difference between the left and right parts of this area is clearly visible by both techniques. These maps allow the exhibition of the spatial variability of the mechanical properties in order to optimize the location of cores.

\section{Conclusions}

The results have been obtained from a two-stage experimental program on an outdoor site-test and a highway bridge. The aim was to analyze the ability of several NDT for assessing the spatial variability of concrete structures

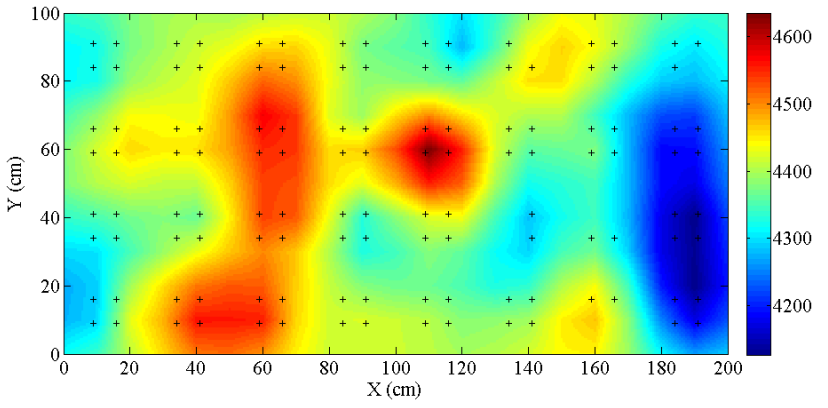

(a)

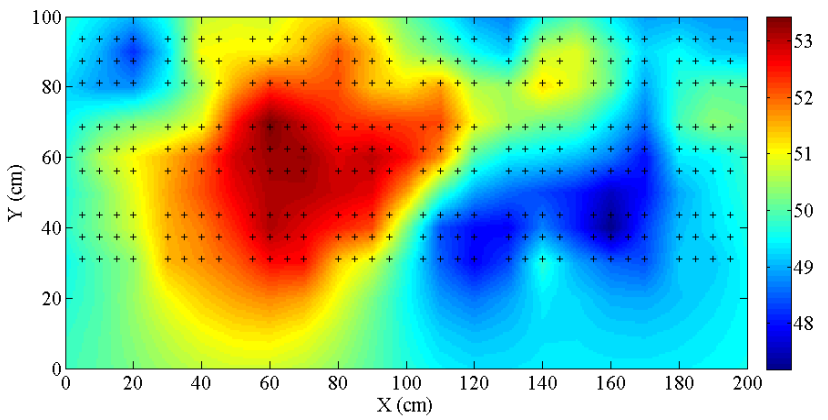

(b)

Fig. 3. Kriging maps of (a) UPV and (b) rebound.

in the real conditions, at different scales: point, local and global. Regarding the magnitude of NDT variability, it is possible to assess the variation of mechanical property (strength) or physical properties (humidity, porosity) by the empirical law. It is also possible to evaluate the minimum number of measurements with an uncertainties level given, at various scales, for each specific survey.

In development, the spatial correlation of NDT measurements is identified by geostatiscal tools. Some correlation length is identified, ranging $40-60 \mathrm{~cm}$ for ultrasonic and electrical resistivity on the limited surface of $2 \mathrm{~m}$ dimensions. This makes it possible to predict the material property of non-sample points by the testing points in which the correlation length. It will be used for optimizing the diagnosis strategy of large structure elements. In consequence, it allows the reduction of the number of measurements without decreasing the evaluation quality for either the sample mean, or the material variability.

\section{References}

[1] D. Breysse, Nondestructive evaluation of concrete strength: An historical review and a new perspective by combining NDT methods, Construct. Build. Mater. 33 (2012) 139-163

[2] A. Aït-Mokhtar et al., Experimental investigation of the variability of concrete durability properties, Cement Concrete Res. 45 (2013) 21-36

[3] J.-P. Balayssac et al., Description of the general outlines of the French project SENSO - Quality assessment and limits of different NDT methods, Construct. Build. Mater. 35 (2012) 131-138 
[4] G. Klysz, J.-P. Balayssac, Determination of volumetric water content of concrete using ground-penetrating radar, Cement Concrete Res. 37 (2007) 1164-1171

[5] M. Saleem et al., Effect of moisture, chloride and sulphate contamination on the electrical resistivity of Portland cement concrete, Construct. Build. Mater. 10 (1996) 209214

[6] Z.M. Sbartaï et al., Using radar direct wave for concrete condition assessment: Correlation with electrical resistivity, J. Appl. Geophys. 62 (2007) 361-374

[7] P.J. Tumidajski et al., On the relationship between porosity and electrical resistivity in cementitious systems, Cement Concrete Res. 26 (1996) 539-544

[8] M.G. Stewart, Q. Suo, Extent of spatially variable corrosion damage as an indicator of strength and timedependent reliability of RC beams, Eng. Struct. 31 (2009) 198-207

[9] F. Schoefs et al., Optimization of non-destructive testing when assessing stationnary processes: application to water and chloride content in concrete, ICDS12, 31 May and 1 June 2012, Lisbon
[10] T. de Larrard et al., Caractérisation expérimentale de la variabilité spatiale des propriétées d'un béton : Identification des longueurs de corrélation et recherche de corrélations entre indicateurs de durabilité, JFMS2012, Chambéry, France, 4-6 juin 2012

[11] N.T. Nguyen et al., Evaluation non destructive des bétons par combinaison des techniques de CND - apport des probabilités conditionnelles, AUGC, Chambéry, France, 4-6 juin 2012

[12] J.-F. Lataste, D. Breysse, A Study on the variability of electrical resistivity of concrete, Nondestructive Testing of Materials and Structures, RILEM 2012, pp. 255-262

[13] P.N. Truong et al., Web-based tool for expert elicitation of the variogram. Comput, Geosci. 51 (2013) 390-399

[14] D. Breysse et al., Quality and accuracy of concrete assessment provided by NDT measurement, 6th International Probabilistic Workshop, Darmstadt, Germany, 26-28 November 2008 